\title{
Perfil nutricional e hábitos alimentares de Policiais Militares
}

\author{
Nutritional profile and food habits of Military Police \\ Perfil nutricional y hábitos alimentarios de la Policía Militar
}

Recebido: 09/09/2021 | Revisado: 15/09/2021 | Aceito: 26/10/2021 | Publicado: 29/10/2021

Helen Mara dos Santos
ORCID: https://orcid.org/0000-0002-6791-6190
Instituto Federal em Educação, Ciência e Tecnologia do Triângulo Mineiro, Brasil
E-mail: helenmara@iftm.edu.br
Estelamar Maria Borges Teixeira
ORCID: https://orcid.org/0000-0002-4486-8966
Instituto Federal em Educação, Ciência e Tecnologia do Triângulo Mineiro, Brasil
E-mail: estelamar@ @iftm.edu.br
Patrícia Maria Vieira
ORCID: https://orcid.org/0000-0002-9963-5465
Universidade Federal do Triângulo Mineiro, Brasil
E-mail: patricia.vieira@uftm.edu.br

\section{Resumo}

As causas do crescente número de casos de sobrepeso e obesidade são multifatoriais, dentre eles podem-se citar fatores genéticos, idade; questões ambientais, emocionais e ocupacionais; e mudanças desfavoráveis na alimentação e atividade física; que podem agir isoladamente ou em conjunto. Este estudo teve por objetivo avaliar o perfil nutricional e os hábitos alimentares de policiais militares na cidade de Uberaba/MG. A coleta dos dados foi realizada com 117 militares, de ambos os sexos, em escala administrativa e operacional. Foram utilizados formulários de autopreenchimento contendo informações das seguintes variáveis: dados socioeconômicos, características clínicas, atividade física, dados antropométricos e questionário de hábitos. Foi avaliado o perfil nutricional conforme as faixas de classificação do Índice de Massa Corporal (IMC) e Circunferência da cintura (CC) para adultos, segundo os parâmetros propostos pela Organização Mundial de Saúde. Observou-se alta frequência de excesso de peso (68\%) entre os policiais militares avaliados e obesidade abdominal com maior prevalência entre o sexo masculino $(71,83 \%)$. Foi observada a correlação positiva entre o IMC e CC. Na distribuição da frequência alimentar, a grande maioria apresentou um baixo consumo de alimentos nutricionalmente adequados. Constatou-se prática de atividade física insuficiente e alimentação não balanceada, que precisam de um cuidado e orientação.

Palavras-chave: Antropometria; Educação alimentar; Hábitos alimentares; Estilo de vida; Polícia Militar.

\begin{abstract}
The causes of the growing number of cases of overweight and obesity are multifactorial, among which we can mention genetic factors, age; environmental, emotional and occupational issues; and unfavorable changes in diet and physical activity; that can act alone or together. This study aimed to assess the nutritional profile and eating habits of military police in the city of Uberaba / MG. Data collection was carried out with 117 military personnel, of both sexes, on an administrative and operational scale. Self-administered forms containing information on the following variables were used: socioeconomic data, clinical characteristics, physical activity, anthropometric data and habits questionnaire. The nutritional profile was evaluated according to the classification ranges of the Body Mass Index (BMI) and Waist circumference (WC) for adults, according to the parameters proposed by the World Health Organization. A high frequency of overweight was observed $(68 \%)$ among the military police officers evaluated and abdominal obesity with a higher prevalence among males $(71.83 \%)$. A positive correlation was observed between BMI and WC. In the distribution of food frequency, the vast majority presented a low consumption of nutritionally adequate foods. Insufficient physical activity and unbalanced diet were found, which need care and guidance.
\end{abstract}

Keywords: Anthropometry; Nutrition education; Eating habits; Lifestyle; Military Police.

\begin{abstract}
o
Las causas del creciente número de casos de sobrepeso y obesidad son multifactoriales, incluidos los factores genéticos, la edad; cuestiones ambientales, emocionales y ocupacionales; y cambios desfavorables en la dieta y la actividad física; que pueden actuar solos o juntos. Este estudio tuvo como objetivo evaluar el perfil nutricional y los hábitos alimentarios de los policías militares de la ciudad de Uberaba / MG. La recolección de datos se realizó con 117 soldados, de ambos sexos, a escala administrativa y operativa. Se utilizaron formularios de autocompletado que contenían información sobre las siguientes variables: datos socioeconómicos, características clínicas, actividad física, datos antropométricos y cuestionario de hábitos. El perfil nutricional se evaluó según los rangos de clasificación de
\end{abstract}


Índice de Masa Corporal (IMC) y Circunferencia de la Cintura (CC) para adultos, según los parámetros propuestos por la Organización Mundial de la Salud. Hubo una alta frecuencia de sobrepeso (68\%) entre los evaluaron policías militares y obesidad abdominal con mayor prevalencia en hombres $(71,83 \%)$. Se observó una correlación positiva entre IMC y CC. En la distribución de la frecuencia alimentaria, la gran mayoría tenía un bajo consumo de alimentos nutricionalmente adecuados. Se constató la práctica de una actividad física insuficiente y una dieta desequilibrada, que necesitan cuidados y orientación.

Palabras clave: Antropometría; Educación nutricional; Hábitos alimenticios; Estilo de vida; Policia Militar.

\section{Introdução}

Considerando a exigência da capacitação física e mental dos policiais tanto para o ingresso como para a permanência nas atividades a fim de cumprir a sua missão. Por estarem envolvidos com a violência e a criminalidade, e desenvolverem atividades nas mais diversas condições, horários, ambientes e situações, as relações internas e externas à corporação, cuja organização se fundamenta na hierarquia rígida e disciplina militar, eles são uma das categorias de trabalhadores que sofrem exposição aos mais variados agravos de saúde, tais como, excesso de peso, baixo nível de atividade física, tabagismo e etilismo (Costa et al., 2007; Donadussi et al., 2009; Grizzle, 2009; Calamita; Silva Filho; Capputti; 2010; Teixeira; Pereira, 2010; Minayo; Assis; Oliveira, 2011; Ferreira; Bonfim; Augusto, 2011; Santana et al., 2012; Barbosa; Silva, 2013; Souza et al., 2013).

O Ministério da Saúde desenvolveu, recentemente, uma pesquisa sobre fatores de risco e proteção para doenças, e demonstrou que a obesidade juntamente com outras doenças crônicas não transmissíveis (DCNT's), foi na última década a primeira causa de morte no Brasil (Brasil, 2017).

As causas do crescente número de casos de sobrepeso e obesidade são multifatoriais, dentre eles podem-se citar fatores genéticos, idade; questões ambientais, emocionais e ocupacionais; e mudanças desfavoráveis na alimentação e atividade física; que podem agir isoladamente ou em conjunto (World Health Organization, 2014; Associação Brasileira para o Estudo da Obesidade e da Síndrome Metabólica, 2016). Esses indivíduos estão predispostos a fatores de riscos, das chamadas DCNT, tais como, doenças cardiovasculares, doenças respiratórias crônicas, doenças metabólicas e diversos tipos de cânceres (World Health Organization, 2014).

Tendo em vista a escassez de estudos com policiais militares e que nenhum estudo com esta temática foi realizado na cidade de Uberaba/Minas Gerais, este estudo tem por objetivo avaliar o perfil nutricional, os hábitos alimentares e estilo de vida de um grupo de policiais militares.

\section{Metodologia}

Trata-se de um estudo de delineamento transversal e observacional, de natureza descritiva, e com abordagem quantitativa. O trabalho foi submetido ao Comitê de Ética em Pesquisa com Seres Humanos (CEPSH) da Universidade Federal do Triângulo Mineiro (UFTM) e autorizado pelo Centro de Pesquisa e Pós Graduação da unidade militar.

A população do estudo compreendeu 117 policiais militares da ativa ou reconvocados, de ambos os sexos, com idade entre 25 a 56 anos, que trabalhavam em escala administrativa ou operacional em uma unidade militar sediada na cidade Uberaba/MG. A participação na pesquisa foi feita de forma voluntária e anônima, mediante a assinatura do Termo de Consentimento Livre e Esclarecido (TCLE).

Foi elaborado um questionário estruturado e auto aplicado para anamnese. Na avaliação antropométrica, os participantes foram submetidos à aferição do peso, altura, Circunferência da Cintura (CC) e cálculo do Índice de Massa Corpórea (IMC). O instrumento utilizado para avaliar os hábitos alimentares, foi teste do Ministério da Saúde, intitulado “Como está sua Alimentação?”, inquérito alimentar auto administrado. Os resultados foram tratados por análises estatísticas (Pereira et al., 2018). 


\section{Resultados}

\subsection{Perfil nutricional}

Participaram do estudo 103 homens (88,0\%) e 14 mulheres (12,0\%), com idade média da idade de 38 anos. Com relação aos dados sociais, demográficos e profissionais dos policiais, a maioria tem escolarização em nível médio (50,43\%) ou superior $(41,03 \%)$, são casados $(74,36 \%)$, atuam em diferentes níveis hierárquicos entre praças $(91,12 \%)$ e oficiais $(8,88 \%)$, com 11 a 20 anos de efetivo serviço na polícia militar(39,56\%), em escala de trabalho predominantemente operacional $(66,00 \%)$.

No relato de história de saúde própria, para o sexo masculino foram os casos de hipertensão arterial sistêmica 11 $(64,7 \%)$, dislipidemias $6(35,2 \%)$, doenças cardiovasculares $3(17,6 \%)$ e17 $(16,5 \%)$, sendo que esse grupo apresenta excesso de peso e gordura visceral, além de muitos possuírem mais de uma doença associada. Na história familiar de DCNT, foram observadas elevadas frequências de hipertensão arterial sistêmica (48,0\%), diabetes mellitus (44,4\%), dislipidemias (28,2\%) e obesidade (22,0\%).

Na Tabela 1, é apresentada a estatística descritiva da idade e as variáveis antropométricas.

Tabela 1. Caracteristicas antropométricas de acordo com o sexo de policiais militares $(n=117)$, da cidade de Uberaba-MG. ${ }^{1}$

\begin{tabular}{|c|c|c|c|c|}
\hline \multirow{3}{*}{$\begin{array}{l}\text { Características } \\
\text { antropométricas }\end{array}$} & \multicolumn{4}{|c|}{ Sexo } \\
\hline & \multicolumn{2}{|c|}{ Feminino $(n=14)$} & \multicolumn{2}{|c|}{ Masculino ( $n=103)$} \\
\hline & Média $\pm D P$ & Variação & Média $\pm D P$ & Variação \\
\hline Idade (anos) & $33,57 \pm 7,35$ & $26,00-50,00$ & $38,67 \pm 6,97$ & $25,00-56,00$ \\
\hline Peso (kg) & $66,17 \pm 9,03$ & $56,50-85,00$ & $84,37 \pm 12,53$ & $52,20-127,00$ \\
\hline Altura $(\mathrm{cm})$ & $1,65 \pm 0,04$ & $1,60-1,75$ & $1,74 \pm 0,05$ & $1,60-1,93$ \\
\hline IMC $\left(\mathrm{kg} / \mathrm{m}^{2}\right)$ & $24,25 \pm 3,28$ & $20,55-28,91$ & $27,70 \pm 3,78$ & $17,74-40,56$ \\
\hline $\begin{array}{l}\text { Circunferência da } \\
\text { cintura }(\mathrm{cm})\end{array}$ & $83,57 \pm 10,03$ & $70,00-105,00$ & $95,79 \pm 10,34$ & $70,00-125,00$ \\
\hline
\end{tabular}

De acordo com o Teste de Significância para igualdade de duas médias (Fonseca; Martins, 1996), a idade, o peso, o IMC e a CC médios são diferentes para o sexo masculino e feminino.

Os homens têm mais idade em relação às mulheres. Em relação ao IMC, as mulheres apresentaram eutrofia e os homens o excesso de peso. Apesar da CC média ter sido maior entre os homens, mulheres e homens apresentam risco aumentado de complicações metabólicas associadas à obesidade.

A altura apresentou baixo desvio padrão entre ambos os sexos, o que reflete homogeneidade entre os policiais militares nesta variável.

Percebeu-se uma alta dispersão de quase todos os dados antropométricos apresentados, em ambos os sexos, o que caracteriza uma grande variação no perfil nutricional dos participantes do estudo.

Com relação à classificação do perfil nutricional dos policiais militares por meio do IMC, somente $31,0 \%$ (n=117) estão com classificação considerada normal ou ideal, $46 \%$ sobrepeso e $19 \%$ obesidade grau I, $2 \%$ obesidade grau II e $1 \%$ obesidade grau III.

De acordo com o sexo, 47,57\% ( $n=49)$ dos homens apresentam sobrepeso e 21,35\% (n=22) com obesidade grau I. Já as mulheres investigadas, $57,14 \%$ (n=8) estão com IMC ideal ou normal e 35,71\%(n=5) com sobrepeso. 
A avaliação da relação do IMC de acordo com a área de atuação dos policiais militares revelou a existência de casos de sobrepeso ou obesidade tanto na área administrativa com $26(63,4 \%)$ policiais quanto na área operacional com $54(71,0 \%)$ policiais. Houve a maior frequência de sobrepeso entre os operacionais e indivíduos com obesidade grau e I e II.

Na Tabela 2 estão descritos os resultados inter-relacionados da classificação da CC com a classificação do IMC, de acordo com o sexo.

Os dados revelam que $26,49 \%$ dos militares com excesso de gordura visceral foram classificados com risco aumentado para complicações metabólicas, sendo quatro mulheres $(28,56 \%)$ e 27 homens $(26,20 \%) ; 29,0 \%$ (n=117) com risco muito aumentado, sendo quatro mulheres $(28,56 \%)$ e 30 homens $(29,11 \%)$.

As mulheres com peso normal, em sua maioria, apresentaram a CC ideal 6 (42,85\%). Em relação aos homens 25 $(24,27 \%)$ estavam com peso normal e a CC ideal. Entre os homens, prevaleceu o sobrepeso e a obesidade 54(52,42\%).

Nota-se que o sobrepeso e obesidade aumentaram com o aumento do risco de complicações metabólicas.

Tabela 2. Relação da classificação da circunferência da cintura pela classificação do índice de Massa Corpórea de acordo com o sexo, de policiais militares $(n=117)$, da cidade de Uberaba-MG. ${ }^{1}$

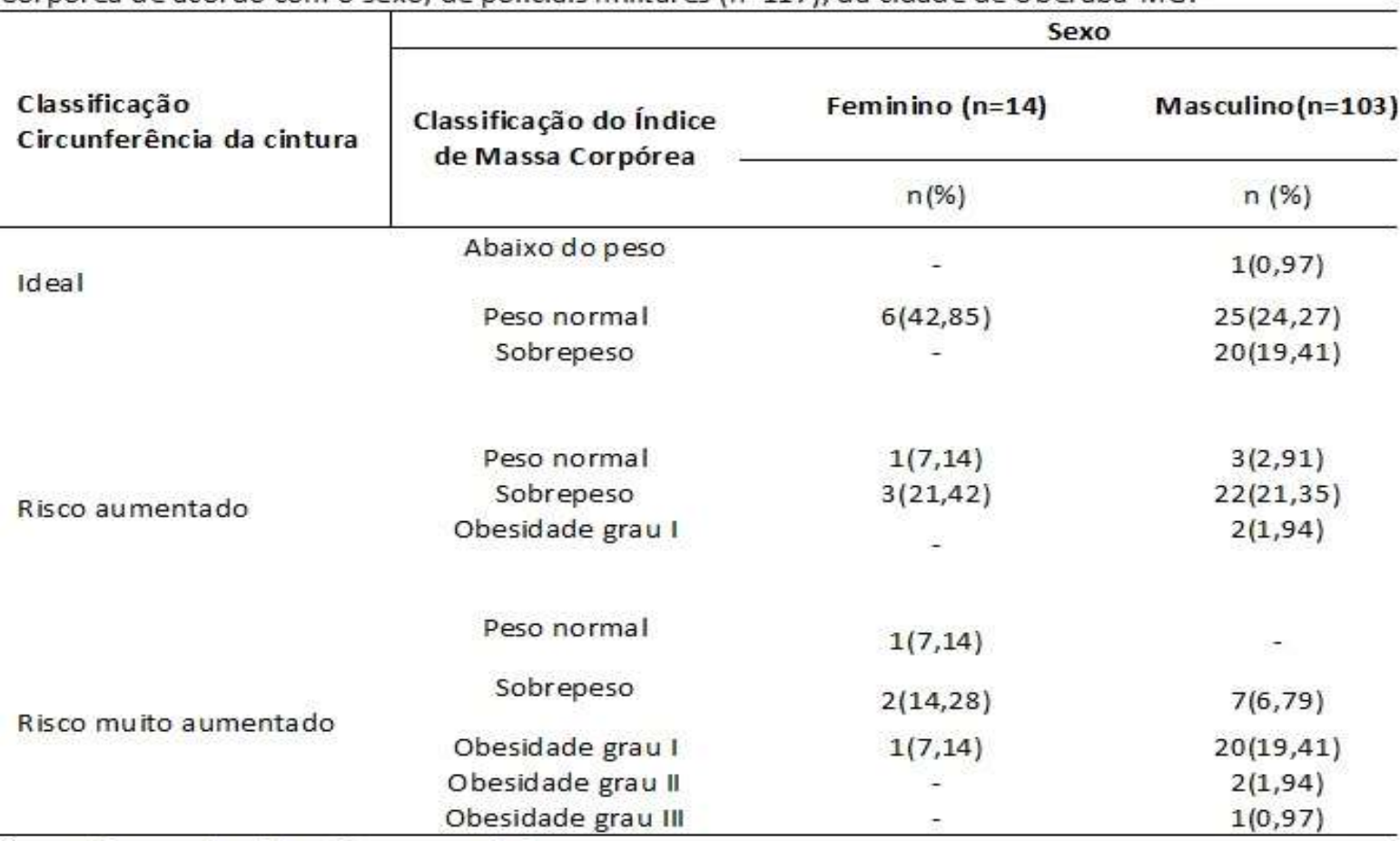

$1_{n}=$ número absoluto e $\%=$ percentual.

Fonte: autoria própria.

Os resultados demonstraram que houve maior frequência de risco aumentado e risco muito aumentado de complicações metabólicas entre o grupo de policiais militares em escala de trabalho operacional, 41 (35,04\%) em comparação com a escala administrativa $24(20,52 \%)$ policiais.

Os diagramas de dispersão demonstraram uma relação linear positiva e forte entre os valores de CC e da classificação do IMC, tanto para o sexo feminino $\left(r=0,8710 ; r^{2}=0,7586 ; \mathrm{p}<0,0001\right)$, quanto para o sexo masculino $\left(\mathrm{r}=0,8450 ; \mathrm{r}^{2}=0,7141\right.$; $\mathrm{p}<0,0001$ ). Pôde-se verificar também o quanto a variação do indicador de obesidade central pode ser predita (ou dependente) em razão da variação nos valores do IMC, em ambos os sexos. 


\subsection{Hábitos alimentares dos policiais militares}

No que se refere à avaliação das práticas alimentares, segundo a classificação do questionário "Como está sua alimentação?" do Ministério da Saúde (2002), observaram-se que 14(12,00\%) policiais precisam tornar a alimentação e seus hábitos de vida mais saudáveis; $87(74,30 \%)$ devem ficar atentos com a alimentação e outros hábitos como atividade física e consumo de líquidos; e 16(13,70\%) estão no caminho para o modo de vida saudável (Tabela 3).

Tabela 3. Classificação do questionário "Como está sua alimentação?"do Ministério da Saúde (2002) de policiais militares ( $n=117$ ), da cidade de Uberaba-MG. ${ }^{1}$

\begin{tabular}{lcc}
\hline “Como está sua alimentação?” & $n$ & Policiais Militares \\
\hline $\begin{array}{l}43 \text { pontos ou mais - Parabéns! Você está no } \\
\text { caminho para o modo de vida saudável. }\end{array}$ & 16 & 13,70 \\
$\begin{array}{l}29 \text { a } 42 \text { pontos - Fique atenta com sua } \\
\text { alimentação e outros hábitos, como atividade }\end{array}$ & 87 & 74,30 \\
física e consumo de líquidos. & & \\
$\begin{array}{l}\text { Até } 28 \text { pontos - Você precisa tornar sua } \\
\text { alimentação e seus hábitos de vida mais } \\
\text { saudáveis. Dê mais atenção a alimentação ea } \\
\text { atividade fisica. }\end{array}$ & 14 & 12,00 \\
\end{tabular}

${ }^{1} n=$ número absoluto $e \%$ = percentual.

Fonte: autoria própria.

Quanto à rotina alimentar semanal, foi relatado pela maioria que as principais refeições são realizadas na residência e com a família (91,5\%); 21,3\% alimentam-se em restaurantes e 0,9\% levam marmita. Sobre a quantidade de refeições habituais no dia, percebe-se o consumo médio de quatro refeições, sendo as mais relatadas o café da manhã $(88,9 \%)$, almoço $(97,4 \%)$, lanche da tarde $(67,5 \%)$ e jantar $(89,7 \%)$.

A ingestão média de frutas por dia para maioria do efetivo entrevistado variou de 1 a 2 unidades/fatias/pedaços/copos de suco natural. Um total de 22 (19\%) relataram que não consomem frutas, nem tomam suco de frutas todos os dias.

Para legumes e verduras, foram poucos os relatos de policiais militares que "não comem legumes, nem verduras todos os dias"7(6\%), sendo o consumo diário da maioria de até 5 colheres de sopa 90(77\%).

A maioria dos policiais militares em função operacional tem a frequência de consumo de frutas delunidade/fatia/pedaço/copo de suco natural correspondendo a $25(32,89 \%)$ e de 3 ou menos colheres de sopa de legumes e verduras por dia, para $33(43,42 \%)$ policiais.

Os policiais militares em função administrativa têm a frequência de consumo de frutas de 2 unidades/fatias/pedaços/copos de suco natural, corresponde $12(29,26 \%)$ policiais e 4 a 5 colheres de sopa de legumes e verduras por dia, para $17(41,46 \%)$ policiais.

Quando se analisa a frequência de consumo de alimentos com alto teor de gordura, açúcar e sal, observa-se que a ingestão desses alimentos pela maioria dos policiais ocorre raramente, nunca ou menos que 2 vezes na semana, 74,3\% para produtos industrializados e $55,5 \%$ para doces em geral. 
Ressalta-se que o grupo das mulheres é mais cauteloso quanto ao consumo de frituras e produtos industrializados de uma forma em geral, prevalecendo50,00\% das respostas para frequência de consumo "raramente ou nunca". E para os doces, tanto mulheres 5(35,71\%) como homens 30(29,10\%), a frequência de consumo foi de "menos que 2 vezes na semana".

Com relação ao consumo de salde adição refinado, foi questionado aos policiais militares participantes da pesquisa se além do sal e/ou tempero contidos nos alimentos preparados, colocavam mais sal no prato, e 85,50\% dos participantes relataram não acrescentar.

Os policiais militares, pertencentes ao grupo de mulheres e homens, em atividade operacional bebem mais água em relação aos que estão em atividade administrativa. Para frequência de consumo de8 copos ou mais por dia, alcançou-se indivíduos 54 (46,15\%). Não houve resposta das mulheres sobre o consumo de 6 a 8 copos de água, sucos e chás.

Foi avaliado o hábito de leitura de policiais militares, de acordo com o sexo, das informações nutricionais nos rótulos de alimentos industrializados para aquisição de produtos alimentícios. Pôde-se verificar que as mulheres demonstraram ser mais preocupadas com essas informações e 50,00\% "algumas vezes, para alguns produtos" ou $28,57 \%$ "sempre ou quase sempre, para todos os produtos" lêem os rótulos de alimentos durante a compra.

\section{Discussão}

As quatro principais DCNT's causadoras de morte no mundo são doença cardiovascular (17,9 milhões de óbitos; 44,0\%); câncer (9,0 milhões de mortes; 22,0\%); doença respiratória crônica (3,8 milhões de mortes; 9,0\%); e diabetes mellitus (1,6 milhões de mortes; 4,0\%) (World Health Organization, 2018). Segundo dados do VIGITEL, em uma década avançaram os casos das DCNT's no Brasil, principalmente o aumento de brasileiros com diagnóstico de diabetes mellitus $(61,8 \%)$ e de hipertensão arterial sistêmica (14,2\%) (Brasil, 2017).

O grupo de homens da presente pesquisa que tem diagnóstico de hipertensão arterial sistêmica, e/ou dislipidemia, e/ou doença cardiovascular que estão associados ainda ao excesso de peso e gordura visceral, merecem monitoramento e intervenção nos fatores de risco. Os resultados dos estudos de Chandra et al., (2014) no Texas/Estados Unidos e de Oliveira et al., (2015) na Bahia/Brasil, corroboram a existência de uma associação entre elevados valores de CC e de hipertensão arterial sistêmica, além de agregar fatores de risco cardiovascular.

Ao analisar a classificação do IMC, medida internacional que avalia o peso de uma pessoa em relação a sua altura e no Brasil, é um dos parâmetros indicados pelo Ministério da Saúde para avaliação do estado nutricional de pessoas (Brasil, 2011) e que tem a sua classificação definida pela OMS (World Health Organization, 2000), foi possível identificar no presente estudo a alta frequência de excesso de peso entre os policiais militares avaliados $(68,0 \%, n=117)$. Quando comparamos os resultados com dados encontrados na pesquisa nacional realizada pelo Ministério da Saúde, em que mais da metade da população brasileira está acima do peso recomendado $(72,7 \%)$ (Brasil, 2017), constatamos que os achados dos policiais estão na média estimada dos padrões nacionais.

O cenário de altas taxas de excesso de peso também é frequente e constante entre militares no Brasil, pois foram encontrados vários estudos nos últimos 10 anos sem diversas regiões do país, utilizando-se o IMC para classificação do perfil nutricional. Nos policiais militares de Cascavel/PR foi encontrada 63,9\% de sobrepeso e obesidade(Donadussi et al., 2009);na cidade do Rio de Janeiro/RJ,48,3\% de sobrepeso e 19,5\% de obesidade (Minayo; Assis; Oliveira, 2011);no Rio Grande do Sul,54,1\% de sobrepeso e 19,8\% de obesidade (Barbosa; Silva,2013); em Floriano/PI,51,0\%de sobrepeso e 23,6\% de obesidade (Neta; Fernandes Filho; Cortez, 2016);em Russas/CE, 62,0\% de sobrepeso e 24,0\% de obesidade(Lima et al.,2016); em Petrolina-PE,51,2\% sobrepeso e 17,6\% obesidade (Lima Júnior et al., 2017) e no Estado de Goiás 80,0\% do efetivo com sobrepeso ou obesidade (Mariano Júnior; Paula, 2018). 
A proporção de policiais militares com obesidade abdominal no presente estudo, $55,6 \% / \mathrm{n}=117$, foi superior aquela relatada para policiais de uma cidade do Sudeste do Brasil (32,1\%) (Santana et al., 2012), no estado da Bahia (31,8\%) (Braga Filho; D'Oliveira, 2013), no Rio Grande do Sul (18,3\%) (Barbosa; Silva, 2013) e na cidade de Cascavel-PR (12,0\%) (DONADUSSI et al.,2009). Achados similares também foram observados na pesquisa realizada com a polícia militar do Estado de Goiás (62,0\%) (Mariano Júnior; Paula, 2018).

O aumento da circunferência abdominal é um dos fatores que estabelece o diagnóstico da Síndrome Metabólica (SM). A SM é definida por transtorno complexo representado por um conjunto de fatores de risco cardiovascular, usualmente relacionados à de posição central de gordura e à resistência à insulina, devendo ser destacada a sua importância do ponto de vista epidemiológico, está associada ao aumento da mortalidade geral em cerca de 1,5 vezes e responsável pelo aumento da mortalidade cardiovascular estimada em 2,5 vezes (Sociedade Brasileira de Cardiologia, 2005).

Em adição a isso, alguns estudos encontraram aumento de peso corporal e da medida da $\mathrm{CC}$ em relação ao tempo de atuação de policiais. Um estudo longitudinal realizado no Departamento de Polícia do Estado da Carolina do Norte nos Estados Unidos, indicou que o aumento dos anos de serviço policial contribuiu para aumentos significativos dos valores da composição corporal, tais como, massa corporal, porcentagem de gordura e massa gorda (Boyce et al., 2008).Da mesma forma, o estudo de Sörensen et al.,(2000), observou aumento no peso e na CC em policiais finlandeses ao longo de 15 anos de trabalho. Salaroli et al., (2016) perceberam que quanto maior o tempo de trabalho dos policiais civis de Vitória/ES, maior a ocorrência de excesso de peso, sendo este mais prevalente entre os indivíduos com mais de 10 anos de profissão $(57,8 \%)$.

Embora no presente estudo tenha-se verificado correlação positiva entre o IMC e CC, nos estudos de Pinho et al., (2012); Machado et al., (2012); Lobato et al., (2014); e Gomes et al., (2015), observaram-se que a obesidade abdominal também está presente em indivíduos com peso normal. Achados de La Scala Teixeira et al., (2017) revelaram que 26,0\% da amostra estudada apresentaram IMC considerado normal e obesidade abdominal. No estudo de Veloso e Silva (2010), feito em seis municípios mais populosos do Estado do Maranhão, observou alta frequência de obesidade abdominal em mulheres com IMC normal.

A obesidade corporal, principalmente em níveis mais altos de valores de IMC, e obesidade abdominal associa-se significativamente a maior mortalidade de todas as causas do que peso normal e contribuem para inúmeras comorbidades (hipertensão arterial sistêmica, dislipidemias, diabetes mellitus, apneia do sono, osteoartrose, doença renal e cardiovascular, câncer, dentre outras),além da incapacidade funcional e redução da qualidade de vida (Associação Brasileira para o Estudo da Obesidade e da Síndrome Metabólica, 2016; Wannmacher, 2016).

Avaliando-se as respostas sobre práticas alimentares dos policiais participantes da pesquisa nota-se que a grande maioria realiza as principais refeições na residência (café da manhã, almoço e jantar). Vale ressaltar que no caso dos policiais em escala operacional, isso acontece nos dias de folga, e os policiais administrativos, com expediente de segunda a sexta-feira, aproveitam a vantagem de morarem em cidade do interior, o que permite tal deslocamento.

O consumo diário de frutas, legumes e verduras foram baixos em relação à recomendação da OMS, que preconiza a ingestão diária de pelo menos 400 gramas de frutas e hortaliças, o que equivale, a aproximadamente, ao consumo diário de cinco porções desses alimentos (World Health Organization, 2003).

Os presentes achados demonstram similaridade com a pesquisa telefônica desenvolvida no Brasil, onde a frequência de adultos que consomem cinco ou mais porções diárias de frutas e hortaliças foi baixa, na maioria das cidades brasileiras estudadas, variando entre 15,5\% em Fortaleza a 32,6\% no Distrito Federal (Brasil, 2017).

Por outro lado, os policiais em atividades operacionais costumam beber mais água, do que os policias em atividade administrava (64,95\%). Salaroli et al., (2016) perceberam em seu estudo menores prevalências de excesso de peso naqueles 
indivíduos que ingeriam 2 litros ou mais de água por dia, em comparação com aqueles que consumiam quantidades inferiores $(62,2 \%)$.

O sódio, encontrado no sal de cozinha (cloreto de sódio) e em grande parte dos alimentos, é um nutriente essencial para o nosso organismo, contribui para o bom funcionamento do corpo. Porém, a ing estão excessiva de sódio está associada ao desenvolvimento da hipertensão arterial sistêmica, doenças renais e outras doenças (Brasil, [2018?]). Em escala mundial, estima-se que a ingestão excessiva de sódio seja por si só, responsável por 1,7 milhões de mortes por doença cardiovascular a cada ano (Organização Pan-Americana da Saúde, 2016). No caso dos policiais, quando questionados sobre o sal de adição nos alimentos já preparados e servidos, a maioria relata não acrescentar.

De modo geral a frequência de consumo pelos policiais militares de frituras, salgadinhos fritos ou de pacote, carnes salgadas, hambúrgueres, presuntos, embutidos, doces de qualquer tipo, refrigerantes e sucos industrializados, foi em sua maioria menor que 2 vezes na semana.

O Guia Alimentar para População Brasileira (2014), publicado pelo Ministério da Saúde, classifica esses alimentos como ultra processados. Os alimentos ultra processados são ricos em gorduras, açúcares e/ou sódio, possuem baixo teor de fibras, o que favorece o consumo excessivo de calorias. A composição nutricional desbalanceada inerente à natureza dos alimentos ultra processados, favorecem o aumento do risco de deficiências nutricionais e a incidência de sobrepeso, obesidade e de DCNT's (Brasil, 2014).

Cavada et al., (2012) avaliaram o hábito de leitura dos rótulos de alimentos de consumidores em uma rede de supermercados de Pelotas-RS, corroborando com os resultados dessa pesquisa. Observaram que a maioria das mulheres são usuárias da rotulagem de produtos no momento da compra de alimentos. Ressalta-se que normalmente é a mulher que cuida e planeja a alimentação da família, fazendo compras e escolhendo os alimentos.

A rotulagem nutricional representa um elo de comunicação entre o consumidor e o produto, pois descreve as propriedades nutricionais de um alimento, compreendendo a declaração de valor energético e os seus principais nutrientes, então, se o rótulo é bem compreendido, permite escolhas alimentares mais criteriosas (Anvisa; UNB, 2005).

\section{Considerações Finais}

Estudos logitudinais são necessários para essa população, para se obter resultados mais robustos e intervenções mais eficazes. uma vez que tais condições aumentam os riscos e/ou pontencializam as doenças crônicas não trasmissiveis e doenças metabólicas que são responsáveis por uma taxa elevada de morbidade e mortalidade quanto não tratadas.

São sugeridos futuros estudos a essa classe de trabalhores, a autoridaes responsaveis devem ter atenção especial no cuidado e manutenção da qualidade do bem-estar físico e mental dos policiais militares.

\section{Agradecimentos}

Agradecemos ao Instituto Federal de Educação, Ciência e Tecnologia do Triângulo Mineiro (IFTM) e a Polícia Militar de Minas Gerais (PMMG).

\section{Referências}

Associação Brasileira para o Estudo da Obesidade e da Síndrome Metabólica. (2016). Diretrizes brasileiras de obesidade 2016. (4a ed.).

Agência Nacional de Vigilância Sanitária - ANVISA, Universidade de Brasília - UnB. (2005). Rotulagem Nutricional Obrigatória: Manual de Orientação às Indústrias de Alimentos. 2. versão. ANVISA, UnB, $44 p$

Barbosa, R. O, Silva, E. F. da. (2013). Prevalência de Fatores de Risco Cardiovascular em Policiais Militares. Rev. BrasCardiol. 26(1), 45-53. 
Boyce, R. W., Jones, G. R., Lloyd, C. L., \& Boone, E. L. (2008). A longitudinal observation of police: body composition changes over 12 years with gender and race comparisons. JEPonline, (11), 1-12.

Braga Filho, R. T., \& D'Oliveira J. A. (2013). The prevalence of metabolic syndrome among soldiers of the military police of Bahia state, Brazil. American Journal of Men's Health, 7(6), 1-6.

Brasil (2011). Ministério da Saúde. Vigilância Alimentar e Nutricional SISVAN. Orientações para a coleta e análise de dados antropométricos em serviços de saúde. http://bvsms.saude.gov.br/bvs/publicacoes/orientacoes_coleta_analise_dados_antropometricos.pdf

Brasil (2014) Ministério da saúde. Secretária de Atenção à Saúde. Departamento de Atenção Básica. Guia alimentar para a população brasileira / Ministério da Saúde, Secretaria de Atenção à Saúde, Departamento de Atenção Básica. (2a ed.), Ministério Da Saúde, 156p. http://portalarquivos.saude.gov.br/images/pdf/2014/novembro/05/Guia-Alimentar-para-a-pop-brasiliera-Miolo-PDF-Internet.pdf

Brasil (2017). Ministério da Saúde. Secretaria de Vigilância em Saúde. Departamento de Vigilância de Doenças e Agravos não Transmissíveis e Promoção da Saúde. Vigitel Brasil 2016: vigilância de fatores de risco e proteção para doenças crônicas por inquérito telefônico: estimativas sobre frequência e distribuição sociodemográfica de fatores de risco e proteção para doenças crônicas nas capitais dos 26 estados brasileiros e no Distrito Federal em 2016 I Ministério da Saúde, Secretaria de Vigilância em Saúde, Departamento de Vigilância de Doenças e Agravos não Transmissíveis e Promoção da Saúde. Brasília: Ministério da Saúde, 160p

Brasil (2018). Ministério da Saúde. Promoção da Saúde e da Alimentação Adequada e Saudável. Sódio - Brasília, DF: Departamento de Atenção Básica. http://dab.saude.gov.br/portaldab/ape_promocao_da_saude.php?conteudo=sodio

Calamita, Z., Silva Filho, C. R., \& Capputti, P. F. (2010). Fatores de risco para doenças cardiovasculares no policial militar. Rev. Bras. Med. Trab. 8(1), 39-45. Cavada, G. S. et al. (2012). Rotulagem nutricional: você sabe o que está comendo? Braz. J. FoodTechnol., IV SSA, 84-88. http://www.scielo.br/pdf/bjft/v15nspe/aop_bjft_15e0115.pdf

Chandra et al. (2014). Fat Distribution and Incident Hypertension. Journal of the American College of Cardiology. 64(10), 997-1002. file:///C:/Users/HELEN/Downloads/997.full.pdf

Costa, M. et al. (2007). Estresse: diagnóstico dos policiais militares em uma cidade brasileira. Revista Panamericana de Salud Pública, 21(4), $217-222$.

Donadussi, C. et al. (2009). Ingestão de lipídios na dieta e indicadores antropométricos de adiposidade em policiais militares. Revista Brasileira de Nutrição, Campinas, 22, (6), 847-855.

Ferreira, D. K. S., Bonfim, C., \& Augusto, L. G. S. (2011). Fatores associados ao estilo de vida de policiais militares. Ciência e Saúde Coletiva, 16(8), 34033412 .

Fonseca, J. S., \& Martins, G. de A. (1996). Curso de Estatística. Ed. Atlas, (6a ed.), 320.

Gomes, et al. (2015). Relação entre Variáveis de Pacientes Cardiopatas. Internacional Journalof Cardiovascular Sciences. .28(5), 392-399.

Grizzle, R. W. (2009). Ocupational Stress, Dietary Self-efficacy, Eating Habits ande Body Composition in Police Officers. Tese de Doutorado. The Universityof Alabama at Birmingham. Birmingham.

La Scala Teixeira, C. V. et al. (2017). Obesidade do peso normal e obesidade abdominal em praticantes de exercício físico: os "falsos magros" existem? Revista Brasileira de Obesidade, Nutrição e Emagrecimento, São Paulo. Suplementar 2.11(68), 748-754. file:///C:/Users/HELEN/Downloads/2017Obesidadedopesonormaleobesidadeabdominalempraticantesdeexercciofsico_osfalsosmagrosexistem.pdf

Lima, A. D. et al. (2016). A associação do índice de massa corpórea com a relação cintura/quadril no comprometimentoda saúde de policiais militares no estado do Ceará. Revista Brasileira de Prescrição e Fisiologia do Exercício, 10(59), 330-339.

Lima Junior et. al., (2017). ARQUIVOS em MOVIMENTO, 13(1), .43-53.

lobato, T. A. A. et al. (2014) Indicadores antropométricos de obesidade em pacientes com infarto agudo do miocárdio. RevBrasCardiol. 27(3), $203-12$.

Machado S. P. et al. (2012). Correlação entre o índice de massa corporal e indicadores antropométricos de obesidade abdominal em portadores de diabetes mellitus tipo 2. RevBrasPromSaude.25(4), 512-20.

Mariano Júnior, D. C., \& Paula, M. A. de. (2018). O risco do sedentarismo ao efetivo da $22^{\circ}$ Companhia independente da Polícia Militar do Estado de Goiás. Revista Brasileira de Estudos de Segurança Pública, 11(1).

Minayo M. C. S, Assis S. G., \& Oliveira R. V. C. (2011). Impacto das atividades profissionais na saúde física e mental dos policiais civis e militares do Rio de Janeiro. Ciênc Saúde Coletiva. 16(4), p.2199-209.

Neta, E. S. A. R., Fernandes Filho, J., \& Cortez, A. C. L. (2016). Nível de atividade física e estado nutricional de policiais militares na cidade de Floriano-PI. Revista Kinesis, 34(1), 84-101.

Oliveira, L. C. de et al. (2015). Prevalência de adiposidade abdominal em adultos de São Francisco do Conde, Bahia, Brasil, 2010. Epidemiol. Serv. Saúde, 24(1), 135-144, http://scielo.iec.gov.br/scielo.php?script=sci_arttext\&pid=S1679-49742015000100015\&lng=pt\&nrm=iso .

OPAS. (2016). Fatores de risco para doenças crônicas não transmissíveis nas Américas: Considerações sobre o fortalecimento da capacidade regulatória. Documento de Referência Técnica REGULA. OPAS.

OPAS (2016). Alimentação e Nutrição: Folhas informativas. Redução do sal. Folha Informativa. https://www.paho.org/bra/index.php?option=co $\mathrm{m} \_$content\&view=article\&id=5439:alimentacao-e-nutricao-folhas-informativas\&Itemid=820 
Research, Society and Development, v. 10, n. 14, e165101420419, 2021

(CC BY 4.0) | ISSN 2525-3409 | DOI: http://dx.doi.org/10.33448/rsd-v10i14.20419

Pereira, A. S., Shitsuka, D. M., Parreira, F. J., \& Shitsuka, R. (2018). Metodologia da pesquisa científica. UFSM. https://repositorio.ufsm.br/bitstrea $\mathrm{m} /$ handle/1/15824/Lic_Computacao_Metodologia-Pesquisa-Cientifica.pdf?sequence=1.

Pinho, P. M. et al. (2012). Correlação entre variáveis nutricionais e clínicas de idosos cardiopatas. RevBrasCardiol. 25(2), 132-40.

Salaroli, L. B. et al. (2016). Indicadores antropométricos e fatores associados: um estudo em policiais civis de Vitória-ES, Brasil. Demetra: alimentação, nutrição \& saúde, 11(4), 1049-1066.

Santana, A. M. C. (2012). Occupational stress, working condition and nutritional status of military police officers. Work, 41 (1), 2908-2914.

Silva, C. P. et al. (2013). Intervenção nutricional pautada na estratégia de oficinas em um serviço de promoção da saúde de Belo Horizonte, Minas Gerais. Revista de Nutrição, 26(6), 647-658.

SBC. (2005). I Diretriz Brasileira de Diagnóstico e Tratamento da Síndrome Metabólica. Arquivos Brasileiros de Cardiologia. 84(I). http://www.scielo.br/pdf/abc/v84s1/a01v84s1.pdf

Souza, E. R. et al. (2013). Consumo de substância lícitas e ilícitas por policiais da cidade do Rio de Janeiro. Ciência e Saúde Coletiva, 18(3), 667-676.

Sörensen, L., Smolander, J., Louhevaara, V., Korhonen, O., \& Oja, P. (2000). Physical activity, fitness and body composition of Finnish police officers: a 15year follow-up study. Occup Med (Lond). 50(1), 3-10.

Teixeira, C. S., \& Pereira, E. F. (2010). Aptidão Física, Idade e Estado Nutricional em Militares. Arq. Bras. Cardiol. 94(4).

Veloso, H. J. F., \& Silva, A. A. M. da. (2010). Prevalência e fatores associados à obesidade abdominal e ao excesso de peso em adultos maranhenses. Rev. bras. epidemiol., 13(3), 400-412.

Wannmacher, L. (2016). Obesidade como fator de risco para morbidade e mortalidade: evidências sobre o manejo com medidas não medicamentosas. 1(7).

WHO. (2000). Obesity: preventing and managing the global epidemic: report of a World Health Organization consultation on obesity. (world health organization technical report series n. 284). Geneva, Switzerland: World Health Organization.

WHO. (2003). Diet, nutrition and the prevention of chronic diseases. Report of a Joint FAO/WHO Consultation. WHO.

WHO. (2014). Global status report on noncommunicable diseases 2014. http://www.fao.org/fileadmin/user_upload/redicean/docs/global\%20status\%20report\%20on\%20NCD.pdf

WHO. (2014). Global status reporton on alcohol and health.Genebra.

WHO. (2018). World health statistics 2018: monitoring health for the SDGs, sustainable development goals. http://apps.who.int/iris/bitstream /handle/10665/272596/9789241565585-eng.pdf?ua=1\&ua=1 